\section{Estudio de factores obstétricos, nutricionales y hormonales y su influencia en el desarrollo de cáncer de mama}

\author{
EMILIO GONZÁLEZ-JIMÉNEZ, MIGUEL ÁNGEL MONTERO-ALONSO², \\ JACQUELINE SCHMIDT RIOVALLE ${ }^{3}$
}

\section{Association between obstetric factors, hormone levels and nutritional status with the development of breast cancer}

Background: Recent studies suggest that the age at first pregnancy, number of children and the lapse between births may protect against breast cancer. Furthermore, serum levels of estrogen, prolactin and progesterone appear to contribute to the development of this tumors in obese women. Aim: To verify whether the variables age at first pregnancy, number of children, birth interval, hormone levels and nutritional status are associated with the age at diagnosis of breast cancer. Material and Methods: Retrospective analysis of medical records of 550 female patients, diagnosed and treated for breast cancer at a hospital in Spain between 2009 and 2012. Results: We found a significant and positive association between age at diagnosis of cancer and the variables age at first pregnancy, parity and interval between pregnancies. There was also a significant correlation $(p<0.000)$ between serum levels of estrogen, prolactin and progesterone and nutritional status of patients. Conclusions: In this sample, age at first pregnancy or number of children, hormone levels and nutritional status are related to the age of onset of cancer.

(Rev Med Chile 2013; 141: 1541-1546)

Key words: Birth intervals; Breast neoplasms; Obesity; Pregnancy; Risk factors.

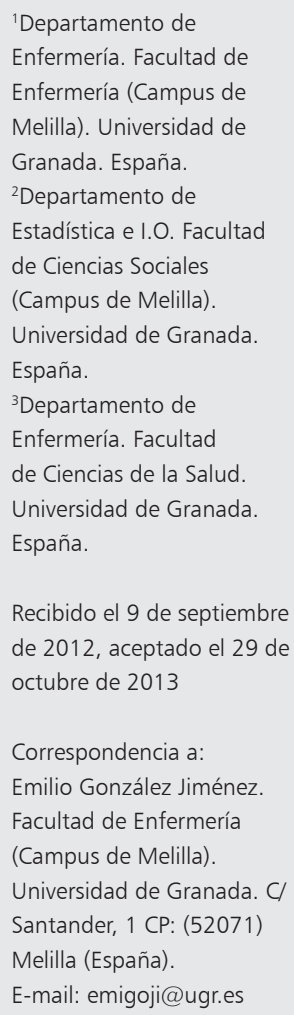

$\mathrm{E}$ 1 cáncer de mama es el principal tipo de cáncer en mujeres en todo el mundo y la causa más común de mortalidad por cáncer entre mujeres de 20 a 59 años de edad ${ }^{1}$. Su incidencia en mujeres está aumentando debido a los cambios en la distribución por edades de la población. Cambios en los patrones de la familia pueden, en cierta medida, explicar el aumento del riesgo real, factores como la obesidad, edad del primer embarazo o el número de hijos parecen ser factores estrechamente relacionados con el desarrollo precoz del cáncer de mama².

Según datos del estudio desarrollado por Haakinson y cols. $(2012)^{3}$, la obesidad en la mujer se encuentra íntimamente relacionada con el desarrollo de tumores mamarios malignos. En este estudio, sus autores concluyen como las pacientes obesas presentan un desarrollo precoz del cáncer de mama así como estadios clínicos más avanzados en el momento de su diagnóstico.

Así, según resultados del estudio desarrollado por Vatten $(1998)^{4}$, aquellas mujeres que dan a luz a su primer hijo a la edad de 30 años poseen doble riesgo de desarrollar un cáncer de mama frente a aquellas otras mujeres que dan a luz a edades de entre 19 y 20 años. Respecto del número de hijos, las mujeres que tenían un único hijo presentaban un riesgo doble de desarrollar tumores mamarios malignos en comparación con las mujeres que habían tenido hasta 4 hijos. En otro importante estudio desarrollado por Magnusson y cols. $(1999)^{5}$ en Suecia a partir de una muestra de 
3.016 mujeres de 50-74 años de edad con cáncer de mama, se concluyó que las mujeres que habían tenido un único hijo desarrollaron un cáncer de mama a edades más tempranas que aquellas otras que habían tenido hasta 5 hijos. Kauppila y cols. $(2012)^{6}$, a partir de una cohorte de 190.949 mujeres finlandesas que habían dado a luz de entre 2 y 4 hijos, muestran como el intervalo de tiempo transcurrido entre el primer nacimiento y el segundo influye fuertemente sobre el riesgo de desarrollar un cáncer de mama. A su vez, esta influencia negativa resultó mayor en aquellas madres que daban a luz después de los 30 años de edad. Es decir, cuando el período de tiempo transcurrido entre el primer nacimiento y el segundo no excedía los dos años en madres menores de 30 años, ello representaba un factor protector previniendo en la madre el desarrollo de un carcinoma mamario.

Ahora bien, en la actualidad existe cierta controversia sobre si los estados de obesidad y obesidad mórbida en la mujer reducen el efecto protector que factores como una edad temprana durante el primer embarazo, tener más de un hijo o un período intergenésico inferior a dos años tienen en la mujer ${ }^{7,8}$.

Otro factor potencialmente implicado en el desarrollo del cáncer de mama en mujeres obesas parece ser los niveles séricos de ciertas hormonas como los estrógenos, prolactina y en menor grado la progesterona. Se cree que los cambios endocrinos secundarios a la obesidad, podrían inducir a cambios sustanciales en el perfil hormonal de estas mujeres ${ }^{9,10}$. Tanto la prolactina como los estrógenos juegan un papel fundamental en el desarrollo normal de la glándula mamaria. Investigaciones recientes han sugerido una asociación entre niveles séricos elevados de estrógenos y prolactina en mujeres obesas y el desarrollo precoz del cáncer de mama ${ }^{11}$. Desde un punto de vista bioquímico, los estrógenos interaccionan con su receptor $(\mathrm{ERa})$ en el núcleo celular. Dicho receptor se une a las regiones reguladoras de sus genes diana, entre los que se encuentran algunos genes implicados en la proliferación celular, favoreciendo así su activación ${ }^{12}$. Por su parte, la prolactina interacciona con su receptor, activándolo a nivel de la membrana celular. Ello facilita la estimulación de vías de señalización proliferativas y anti-apoptóticas en la célula tumoral ${ }^{13}$.

Teniendo en cuenta que tras la menopausia los ovarios dejan de producir estrógenos, será el tejido adiposo quien se convierta en la principal fuente de esta hormona ${ }^{14}$. En este sentido, tejidos como el parénquima mamario muy sensibles a los estrógenos, quedarán expuestos a un mayor estímulo hormonal en mujeres obesas ${ }^{15,16}$. Considerando todo lo anterior, los objetivos en este trabajo fueron verificar si las variables edad del primer embarazo, número de hijos y período de tiempo intergenésico ejercían un efecto protector en la mujer mediante una edad de diagnóstico más tardía del cáncer de mama. En segundo lugar comprobar la existencia de una relación significativa entre el estado nutricional de las pacientes y sus niveles séricos de estrógenos, prolactina y progesterona.

\section{Objetivos}

Este estudio tiene como objetivos confirmar si las variables edad del primer embarazo, número de hijos y período de tiempo intergenésico ejercen un efecto protector en la mujer mediante una edad de diagnóstico más tardía del cáncer de mama. En segundo lugar, comprobar la existencia de una relación significativa entre los niveles séricos de estrógenos, prolactina y progesterona y el estado nutricional de las pacientes.

\section{Muestra}

La muestra estaba compuesta por 550 pacientes de sexo femenino, diagnosticadas y tratadas de cáncer de mama en el Hospital Universitario Virgen de las Nieves de Granada (España), durante el período de enero de 2009 a enero de 2012.

\section{Metodología}

Este estudio utilizó un diseño basado en una recogida retrospectiva de datos clínicos de todas y cada una de las pacientes por medio de una revisión minuciosa de su historia clínica. Para el acceso al historial clínico fue necesario contar previamente con la autorización por escrito de cada paciente o su representante legal, cumpliendo así con los principios fundamentales sobre investigación con seres humanos recogidos en la Declaración de Helsinki: (autorización para el acceso a las historias clínicas, protección de datos personales y confidencialidad absoluta de los datos recabados). El análisis de los datos fue realizado con el programa informático SPSS, versión 15 para Windows. 
Factores obstétricos, nutricionales y hormonales en cáncer mamario - E. González et al

\section{Resultados}

El análisis descriptivo de los datos muestra que la edad media a la que se diagnostica el cáncer de mama es aproximadamente 57 años y medio, en un abanico entre 19 y 91 años, aunque la edad más frecuente es la de 65 años. Destacar que sólo $4,18 \%$ de las mujeres estudiadas no han tenido hijos, donde el número medio y más frecuente es de 3 hijos. La Tabla 1 muestra las características descriptivas más significativas de las variables estudiadas.

El análisis de regresión múltiple tomando como variable dependiente la edad de diagnóstico y como variables independientes el período intergenésico (medido en meses), el número de hijos y la edad en el primer embarazo confirma niveles de significación para todos coeficientes, con un nivel crítico o p-valor de 0,000 , lo que indica que es significativamente distinto de cero. Estos resultados se muestran más claramente en la Tabla 2.

El modelo ajustado presenta un coeficiente de determinación de 0,886 . Ello indica que $88,6 \%$ de la variabilidad en la edad de diagnóstico del cáncer de mama se justifica por su relación lineal con las tres variables edad del primer embarazo, número de hijos y período de tiempo intergenésico.

En la Tabla 3 se muestra como los coeficientes del modelo ajustado son estadísticamente significativos $(\mathrm{p}<0,000)$ y un valor del coeficiente de correlación $r=0,941$ (Tabla 4), lo que denota una fuerte asociación lineal positiva entre la edad de diagnóstico y las variables edad del primer embarazo, número de hijos y período intergenésico.

Respecto de la posible relación entre el estado nutricional de las pacientes y sus niveles

Tabla 1. Análisis descriptivo de las variables analizadas

\begin{tabular}{|c|c|c|c|c|c|}
\hline & & $\begin{array}{c}\text { Edad } \\
\text { diagnóstico }\end{array}$ & $\begin{array}{l}\text { Edad primer } \\
\text { embarazo }\end{array}$ & n de hijos & $\begin{array}{c}\text { Período intergenésico } \\
\text { (meses) }\end{array}$ \\
\hline \multirow[t]{2}{*}{$n$} & Válidos & 550 & 527 & 550 & 550 \\
\hline & Perdidos & 0 & 23 & 0 & 0 \\
\hline Media & & 57,57 & 23,20 & 3,01 & 11,01 \\
\hline Mediana & & 58,00 & 23,00 & 3,00 & 10,00 \\
\hline Moda & & 65 & 25 & 3 & 0 \\
\hline Desv. típ. & & 15,245 & 3,740 & 1,521 & 7,904 \\
\hline Asimetría & &,- 088 &,- 121 & ,410 & 1,749 \\
\hline Curtosis & &,- 849 & 1,975 & ,463 & 5,319 \\
\hline Mínimo & & 19 & 0 & 0 & 0 \\
\hline Máximo & & 91 & 34 & 8 & 48 \\
\hline \multirow[t]{2}{*}{ Percentiles } & 2,5 & 29,00 & 17,20 & ,00 & ,00 \\
\hline & 97,5 & 85,00 & 31,00 & 6,00 & 36,00 \\
\hline
\end{tabular}

Tabla 2. ANOVA

\begin{tabular}{|c|c|c|c|c|c|c|}
\hline Modelo & & Suma de cuadrados & gl & Media cuadrática & $\mathbf{F}$ & Sig. \\
\hline \multirow{3}{*}{1} & Regresión & 96358,462 & 3 & 32119,487 & 1348,946 &, $000^{\circ}$ \\
\hline & Residual & 12429,241 & 522 & 23,811 & & \\
\hline & Total & 108787,703 & 525 & & & \\
\hline
\end{tabular}

aVariables predictoras: (Constante), Período Intergenésico (meses), Número de hijos, Edad Primer Embarazo. bVariable dependiente: Edad Diagnóstico. 
Factores obstétricos, nutricionales y hormonales en cáncer mamario - E. González et al

Tabla 3. Coeficientes del modelo ajustado

\begin{tabular}{|lccccc|}
\hline Modelo & \multicolumn{2}{c}{$\begin{array}{c}\text { Coeficientes } \\
\text { no estandarizados } \\
\text { Error típ. }\end{array}$} & $\begin{array}{c}\text { Coeficientes } \\
\text { estandarizados } \\
\text { Beta }\end{array}$ & t & Sig. \\
(Constante) & 113,197 & 2,903 & & 38,987 &, 000 \\
Edad primer embarazo & $-2,719$ &, 099 &,- 681 & $-27,511$ &, 000 \\
Número de hijos & 3,038 &, 247 &, 299 & 12,292 &, 000 \\
Período Intergenésico (meses) &,- 065 &, 028 &,- 035 & $-2,286$ &, 023 \\
\hline
\end{tabular}

`ariable dependiente: Edad diagnóstico.

Tabla 4. Resumen del modelo

\begin{tabular}{|ccccc|}
\hline Modelo & R & R cuadrado & R cuadrado corregida & Error típ. de estimación \\
\hline 1 & 0,941 & 0,886 & 0,885 & 4,880 \\
\hline
\end{tabular}

Tabla 5. Estimaciones de los parámetros

\begin{tabular}{|lcccccccc|}
\hline Obesidada & B & $\begin{array}{c}\text { Error } \\
\text { típ. }\end{array}$ & & Wald & gl & Sig. & Exp (B) & $\begin{array}{c}\text { Intervalo de confianza a 95\% } \\
\text { para Exp (B) } \\
\text { Límite } \\
\text { Límite } \\
\text { superior }\end{array}$ \\
\hline Estradiol & 0,823 & 0,112 & 54,348 & 1 & 0,000 & 2,277 & 1,830 & 2,834 \\
Prolactina & $-0,656$ & 0,105 & 39,241 & 1 & 0,000 & 0,519 & 0,423 & 0,637 \\
\hline Progesterona & $-10,181$ & 3,071 & 10,991 & 1 & 0,001 & 0,0000379 & $9,21 \mathrm{E}-08$ & 0,016 \\
\hline
\end{tabular}

aLa categoría de referencia es: 1.

séricos de estradiol, prolactina y progesterona, los datos muestran como a medida que el peso de las pacientes se eleva, los niveles séricos de dichas hormonas se incrementan paralelamente. Para dicho estudio se ha realizado una Regresión Logística Multivariante, donde los resultados obtenidos (Tabla 5) revelan que la fracción de mujeres con sobrepeso frente a las de peso normal es 2,277 veces mayor para aquellas mujeres que poseen niveles de estradiol elevados frente a aquellas otras que no los tienen. Del mismo modo, la fracción de mujeres con sobrepeso frente a las de peso normal es 0,519 veces mayor para aquellas otras mujeres que poseen niveles de prolactina elevados y 0,0000379 veces mayor para las mujeres que poseen niveles elevados de progesterona. Considerando que los valores de las Odd Ratio están debidamente ajustados, se elimina la posibilidad de que un factor confunda el efecto de otro y siendo todos significativos.

\section{Discusión}

Los resultados obtenidos en este estudio confirman la existencia de una relación lineal entre la edad del diagnóstico del cáncer y las variables edad del primer embarazo, número de hijos y período de tiempo intergenésico. De acuerdo con Veronesi $(2005)^{17}$, de las tres variables estudiadas, tener el primer embarazo antes de los 30 años, así como haber dado a luz más de un hijo constituyen los factores más directamente relacionados con una edad de diagnóstico de cáncer de mama más tardía. Estos resultados concuerdan con los reportados por otros estudios como el desarrollado 
por Phillips y cols. (2009) ${ }^{18}$. Luego los resultados alcanzados, resultan preocupantes si tenemos en cuenta que, en la actualidad, la edad del primer embarazo en la mujer española se ha retrasado considerablemente, situándose próxima a los 30 años ${ }^{19}$. Del mismo modo, el promedio de hijos por cada pareja en España se sitúa en $1,2^{19}$.

Respecto a la relación existente entre los niveles séricos de estrógenos, prolactina y el estado nutricional de las pacientes cabe destacar como a medida que las pacientes incrementan su peso, los niveles circulantes de dichas hormonas se elevan.

Ello constituye un factor de riesgo importante si consideramos que ciertas hormonas como los estrógenos se aromatizan en el tejido graso incrementándose sus niveles circulantes. Luego, de acuerdo con Parkin $(2011)^{20}$, Lonning $(2011)^{21}$ y Fernández $(2010)^{22}$, tejidos eminentemente grasos como el parénquima mamario y por ende, muy sensibles a los estrógenos, quedarán expuestos a un mayor estímulo hormonal en mujeres con sobrepeso y obesas. En el caso de la progesterona, los resultados obtenidos muestran una correlación menor respecto del estado nutricional entre las pacientes estudiadas, circunstancia que coincide con lo descrito por otros autores en estudios previos $^{23,24}$.

A tenor de los resultados alcanzados, cabe concluir cómo para la población de mujeres estudiada, factores como la edad del primer embarazo o el número de hijos tenidos suponen factores íntimamente relacionados con la edad de aparición del cáncer. Del mismo modo, el estado nutricional parece constituir otro factor implicado en la génesis del cáncer de mama, por su relación directa con hormonas (estrógenos y prolactina). En cualquier caso, serán necesarios nuevos estudios en los que se profundicen las implicaciones que las variables estudiadas poseen sobre el desarrollo del cáncer de mama.

\section{Referencias}

1. Warren Andersen S, Newcomb PA, Hampton JM, TitusErnstoff L, Egan KM, Trentham-Dietz A. Reproductive factors and histologic subtype in relation to mortality after a breast cancer diagnosis. Breast Cancer Res Treat 2011; 130 (3): 975-80.

2. Lord SJ, Bernstein L, Johnson KA, Malone KE, McDonald JA, Marchbanks PA, et al. Breast cancer and hormo- ne receptor status in older women by parity, age at first birth, and breastfeeding: a case control study. Cancer Epidemiol Biomarkers Prev 2008; 17: 1723-30.

3. Haakinson DJ, Leeds SG, Dueck AC, Gray RJ, Wasif N, Stucky CC, et al. The Impact of Obesity on Breast Cancer: A Retrospective Review. Ann Surg Oncol 2012; 27 [Epub ahead of print].

4. Vatten L. Female sex hormones increase the risk of breast cancer. Tidsskr Nor Laegeforen 1998; 118 (19): 2969-74.

5. Magnusson CM, Persson IR, Baron JA, Ekbom A, Bergström R, Adami HO. The role of reproductive factors and use of oral contraceptives in the aetiology of breast cancer in women aged 50 to 74 years. Int J Cancer 1999; 80 (2): 231-6.

6. Kauppila A, Kyyrönen P, Lehtinen M, Pukkala E. Dual effect of short interval between first and second birth on ductal breast cancer risk in Finland. Cancer Causes Control 2012; 23 (1): 187-93.

7. Almquist M, Bondeson AG, Bondeson L, Halthur C, Malm J, Manjer J. Reproductive history, lifestyle factors and season as determinants for serum calcium concentrations in women. Scand J Clin Lab Invest 2008; 68 (8): 777-85.

8. Tsuji M, Tamai Y, Wada K, Nakamura K, Hayashi M, Takeda N, et al. Associations of intakes of fat, dietary fiber, soy isoflavones, and alcohol with levels of sex hormones and prolactin in premenopausal Japanese women. Cancer Causes Control 2012; 8 [Epub ahead of print].

9. Hines LM, Risendal B, Slattery ML, Baumgartner KB, Giuliano AR, Sweeney C. Comparative analysis of breast cancer risk factors among hispanic and non-hispanic white women. Cancer 2010; 1: 3215-23.

10. Key TJ, Appleby PN, Reeves GK, Roddam AW, Helzlsouer KJ, Alberg AJ, et al. Circulating sex hormones and breast cancer risk factors in postmenopausal women: reanalysis of 13 studies. British Journal Cancer 2011; 105 (5): 709-22.

11. Key TJ. Endogenous oestrogens and breast cancer risk in premenopausal and postmenopausal women. Steroids 2011; 76 (8): 812-5.

12. McTiernan A, Wu L, Barnabei VM, Chen C, Hendrix S, Modugno F, et al; WHI Investigators. Relation of demographic factors, menstrual history, reproduction and medication use to sex hormone levels in postmenopausal women. Breast Cancer Res Treat 2008; 108 (2): 217-31.

13. Secreto G, Meneghini E, Venturelli E, Cogliati P, Agresti R, Ferraris C, et al. Circulating sex hormones and tumor characteristics in postmenopausal breast cancer patients. 
A cross-sectional study. Int J Biol Markers 2011; 26 (4): 241-6.

14. Li XJ, Wei B, Chen HJ, Zhang Z, Zhang HY, Bu H. Heat-induced antigen retrieval in inmunohistochemistry for estrogen and progesterone receptors in breast cancer tissues. Zhonghua Bing Li Xue Za Zhi 2011; 40 (6): 406-8.

15. Beral V. Breast cancer and hormone-replacement therapy in the Million Women Study. Lancet 2003; 362 (9382): 419-27.

16. Faupel-Badger JM, Sherman ME, García Closas M, Gaudet MM, Falk RT, Andaya A, et al. Prolactin serm levels and breast cancer: relationships with factors and tumour characteristics among pre- and postmenopausal women in a population-based case-control study from Poland. British Journal Cancer 2010; 103 (7): 1097-102.

17. Veronesi U, Boyle P, Goldhirsch A, Orecchia R, Viale G. Breast cancer. Lancet 2005; 365 (9472): 1727-41.

18. Phillips KA, Milne RL, West DW, Goodwin PJ, Giles GG, Chang ET, et al. Prediagnosis reproductive factors and all-cause mortality for women with breast cancer in the breast cancer family registry. Cancer Epidemiol Biomarkers Prev 2009; 18 (6): 1792-7.

19. Peñacoba-Puente C, Monge FJ, Morales DM. Pregnancy worries: a longitudinal study of Spanish women. Acta Obstet Gynecol Scand 2011; 90 (9): 1030-5.

20. Parkin DM. Cancers attributable to exposure to hormones in the UK in 2010. British Journal of Cancer 2011; 105: $42-8$

21. Lonning PE, Haynes BP, Straume AH, Dunbier A, Helle $\mathrm{H}$, Knappskog S, et al. Exploring breast cancer estrogen disposition: the basis for endocrine manipulation. Clin Cancer Research 2011; 17 (15): 4948-58.

22. Fernández I, Touraine P, Goffin V. Prolactin and human tumourogenesis. J Neuroendocrinol 2010; 22 (7): 771-7.

23. Lange CA, Yee D. Progesterone and breast cancer. Womens Health 2008; 4 (2): 151-62.

24. Yoshimoto N, Nishiyama T, Toyama T, Takahashi S, Shiraki N, Sugiura H, et al. Genetic and environmental predictors, endogenous, hormones and growth factors, and risk of estrogen receptor positive breast cancer in Japanese women. Cancer Sci 2011; 26 [Epub ahead of print]. 